\title{
An Inverse Scattering Approach Based on the Field Equivalence Principle: Inversion without a-Priori Information on Incident Fields
}

\author{
Takashi Takenaka* and Toshifumi Moriyama \\ Department of Electrical and Electronic Engineering, Graduate School of Engineering, \\ Nagasaki University, 1-14 Bunkyo-machi, Nagasaki 852-8521, Japan \\ *Corresponding author: takenaka@nagasaki-u.ac.jp
}

Received Month X, XXXX; revised Month X, XXXX; accepted Month X, XXXX; posted Month X, XXXX (Doc. ID XXXXX); published Month X, XXXX

\begin{abstract}
An inverse scattering approach based on the field equivalence principle is developed for reconstructing medium parameters of a scattering object. A problem equivalent to the original scattering problem internal to the measurement surface is set up. The equivalent surface currents determined by the measured total field data yield the rigorous incident fields in the region under test so that the approach does not need explicit knowledge of the incident fields. Taking into account the fact that the equivalent surface currents produce a null field external to the surface, a functional of the medium parameters is introduced and a genetic algorithm is applied to the minimization of the functional. Numerical simulations for imaging defects in a known dielectric cylinder from only total field data measured on an observation surface are performed to illustrate the efficacy of the proposed inversion method. (C) 2010 Optical Society of America

OCIS Codes: 000.0000, 999.9999
\end{abstract}

A variety of inverse scattering methods have been proposed for estimating the electrical parameter distributions of an unknown object from the total field measured on an observation surface last decades [1-16]. The explicit knowledge of the incident field is often assumed. Only measured total field data is, however sufficient for inversion [17].

In this paper, we propose a novel inverse scattering method for reconstructing electrical parameters of inhomogeneous objects from only measured total field data without explicitly using a-priori information regarding the incident field. The basic idea of the proposed method is based on the field equivalence principle [18]. Whatever the primary source producing fields illuminating unknown objects is, the incident field in the estimated region is calculated accurately by the equivalent surface current (related to the tangential components of the total field) on an observation surface containing the estimated region if the source is located outside the observation surface.

Let us consider a scattering problem by an inhomogeneous object embedded in a homogeneous background medium with the relative permittivity $\varepsilon_{b}$, permeability $\mu_{b}$, and conductivity $\sigma_{b}$ as shown in Fig. 1(a). The short pulsed wave is generated by an electric current source $\mathbf{J}(\mathbf{r}, t)$ where $\mathbf{r}=(x, y, z)$. We assume that the transmitter source is turned on at time $t=0$ and there is no electromagnetic fields before time $t=0$. Then, the total electromagnetic fields $\mathbf{v}(\mathbf{r}, t)$ satisfy Maxwell's equations in the following matrix form:

$$
\mathcal{L} \mathbf{v}=\mathbf{j}
$$

where

$$
\begin{gathered}
\boldsymbol{v}=\left(E_{x}, E_{y}, E_{z}, \eta H_{x}, \eta H_{y}, \eta H_{z}\right)^{\mathrm{t}} \\
\mathbf{j}=\left[\eta J_{x}, \eta J_{y}, \eta J_{z}, 0,0,0\right]^{\mathrm{t}}
\end{gathered}
$$

under the initial condition of zero fields:

$$
\mathbf{v}(\mathbf{r}, t)=\mathbf{0}, \quad t<0
$$

The superscript ' $\mathrm{t}$ ' indicates 'transposed'. $\eta\left(=\sqrt{\mu_{0} / \varepsilon_{0}}\right)$ is the characteristic impedance of free space. The partial differential operator $\mathcal{L}$ is defined by

$$
\mathcal{L} \equiv \bar{A} \frac{\partial}{\partial x}+\bar{B} \frac{\partial}{\partial y}+\bar{C} \frac{\partial}{\partial z}-\bar{F} \frac{\partial}{\partial(c t)}-\bar{G}
$$

where $c\left(=1 / \sqrt{\varepsilon_{0} \mu_{0}}\right)$ is the speed of light in free space, $\bar{A}$, $\bar{B}, \bar{C}$ are $6 \times 6$ constant matrices whose components are $A_{26}=-A_{35}=-A_{53}=A_{62}=1 \quad, \quad B_{16}=-B_{34}=-B_{43}=B_{61}=1 \quad$, $C_{15}=-C_{24}=-C_{42}=C_{51}=-1, A_{m n}=B_{m n}=C_{m n}=0 \quad$ otherwise, and

$$
\bar{F}=\left[\begin{array}{cc}
\varepsilon_{r} \bar{I} & \mathbf{0} \\
\mathbf{0} & \mu_{r} \bar{I}
\end{array}\right], \quad \bar{G}=\left[\begin{array}{cc}
\eta \sigma \bar{I} & \mathbf{0} \\
\mathbf{0} & \mathbf{0}
\end{array}\right]
$$

and where $\varepsilon_{r}, \mu_{r}$, and $\sigma$ are the relative permittivity, relative permeability, and conductivity of the medium, respectively. $\bar{I}$ denotes the $3 \times 3$ identity matrix.

Let us consider two regions, denoted by $\Omega$ and $\bar{\Omega}$, and separated by an observation surface $\partial \Omega$. The impressed source $\mathbf{J}$ is assumed to be in the region $\bar{\Omega}$ while the scattering object in the region $\Omega$. Let us set up a problem equivalent to the original problem internal to $\partial \Omega$, i.e., the original field exists in $\Omega$ and the null field in $\bar{\Omega}$. In the equivalent problem, the original source $\mathbf{J}$ (and obstacles if any) is removed and equivalent sources are placed on the 


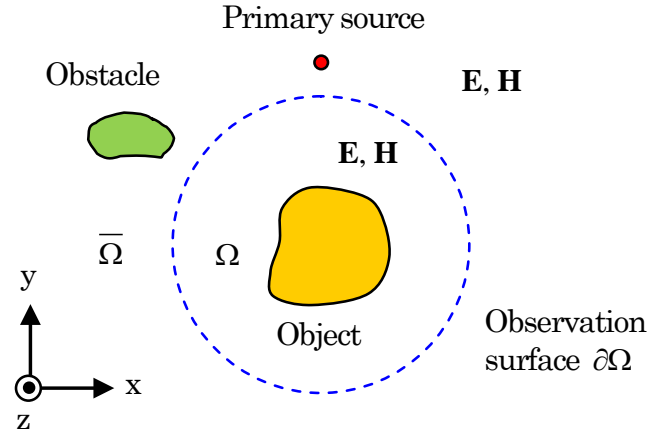

(a)

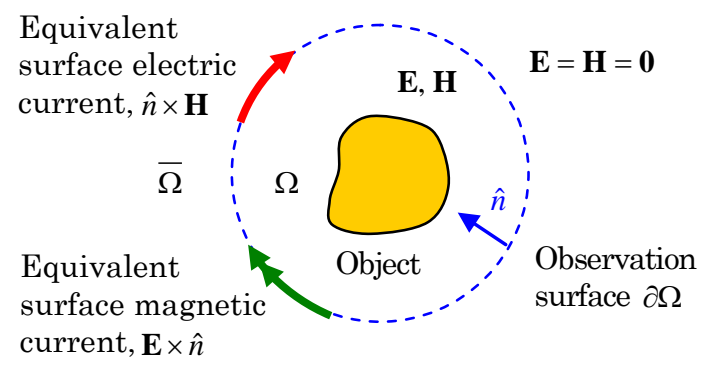

(b)

Fig. 1 (a) Original and (b) equivalent problems for the interior region $\Omega$.

observation surface $\partial \Omega$ as shown in Fig. 1(b). In order to reproduce the same field internal to $\partial \Omega$ as do the original source and the null field external to $\partial \Omega$, the surface electric and magnetic currents, $\mathbf{J}_{s}=\hat{n} \times \mathbf{H}, \mathbf{M}_{s}=\mathbf{E} \times \hat{n}$ are placed on $\partial \Omega$ where $(\mathbf{E}, \mathbf{H})$ are the original total fields over $\partial \Omega$. The total fields $(\mathbf{E}, \mathbf{H})$ are the sum of the incident fields $\left(\mathbf{E}^{\text {inc }}, \mathbf{H}^{\text {inc }}\right)$ and scattered fields $\left(\mathbf{E}^{\text {sca }}, \mathbf{H}^{\text {sca }}\right)$. The unit vector $\hat{n}$ is inward normal to $\partial \Omega$.

Electromagnetic fields of the equivalent problem are the solution of the following Maxwell's equations:

$$
\mathcal{L} \mathbf{u}=\mathbf{s}
$$

under the initial condition of zero fields:

$$
\mathbf{u}(\mathbf{r}, t)=\mathbf{0}, \quad t<0
$$

where

$$
\begin{gathered}
\mathbf{u}=\left(E_{x}^{e q}, E_{y}^{e q}, E_{z}^{e q}, \eta H_{x}^{e q}, \eta H_{y}^{e q}, \eta H_{z}^{e q}\right)^{\mathrm{t}} \\
\mathbf{s}=\left(\begin{array}{c}
s_{1} \\
s_{2} \\
s_{3} \\
s_{4} \\
s_{5} \\
s_{6}
\end{array}\right)=\left(\begin{array}{c}
n_{y} \eta H_{z}-n_{z} \eta H_{y} \\
n_{z} \eta H_{x}-n_{x} \eta H_{z} \\
n_{x} \eta H_{y}-n_{y} \eta H_{x} \\
-n_{y} E_{z}+n_{z} E_{y} \\
-n_{z} E_{x}+n_{x} E_{z} \\
-n_{x} E_{y}+n_{y} E_{x}
\end{array}\right) \delta_{\partial \Omega}
\end{gathered}
$$

and where $\delta_{\partial \Omega}$ is the delta function representing a source concentrated on the surface $\partial \Omega$. The source $\mathbf{s}$ produces the same total fields as do the original source internal to $\partial \Omega$ while the null field external to $\partial \Omega$, i.e.,

$$
\mathbf{u}(\mathbf{r}, t)=\left\{\begin{array}{cc}
\mathbf{v}(\mathbf{r}, t) & \mathbf{r} \in \Omega \\
\mathbf{0} & \mathbf{r} \in \bar{\Omega}
\end{array}\right.
$$

Note that the component $\mathbf{s}^{s c a}$ of the source $\mathbf{s}\left(=\mathbf{s}^{i n c}+\mathbf{s}^{\text {sca }}\right)$ related to the scattered field by the object reproduces faithfully the negative of the scattered field propagating outward from $\partial \Omega$ and does not contribute to fields in $\Omega$. The component $\mathbf{s}^{\text {inc }}$ of $\mathbf{s}$ corresponding to the incident field reproduces faithfully the incident field in $\Omega$ which is scattered by the object, so that the source $\mathbf{s}^{\text {inc }}$ contributes to producing the scattered field over the whole region $\Omega \cup \bar{\Omega}$. In the region $\bar{\Omega}$ the negative of the scattered field produced by the source $\mathbf{s}^{\text {sca }}$ cancels out completely the scattered field due to the source $\mathbf{s}^{\text {inc }}$ and the object.

Suppose transient total field data are measured on the observation surface $\partial \Omega$ during a time interval $[0, T]$. The time-domain inverse scattering problem under consideration here is the estimation of $\varepsilon_{r}(\mathbf{r}), \mu_{r}(\mathbf{r})$, and $\sigma(\mathbf{r})$ with the knowledge of the tangential components of the original total fields $\mathbf{v}(\mathbf{r}, t)$ measured on the surface $\partial \Omega$ but without the explicit knowledge of the incident field. According to the equivalence principle, when the estimated electrical parameters are identical with the true ones, the fields produced by the equivalent surface current $\mathbf{s}$ cancel out the scattered fields in the region $\bar{\Omega}$ by the object so that no fields are there during the time interval $[0, T]$. Incorrectly estimated parameters, however, do not yield null fields in the region $\bar{\Omega}$. Based on this observation, we formulate the inverse problem as an optimization problem in which the following cost functional needs to be minimized:

$$
Q(\mathbf{p})=\sum_{n=1}^{N} \int_{0}^{c T} \int_{\bar{\Omega}}\left|\mathbf{u}_{n}(\mathbf{p} ; \mathbf{r}, t)\right|^{2} d \mathbf{r} d(c t)
$$

where $\mathbf{p}=\left[\varepsilon_{r}(\mathbf{r}), \mu_{r}(\mathbf{r}), \eta \sigma(\mathbf{r})\right]$ is an electrical parameter vector of the object. $N$ successive illuminations are assumed to probe the object. The vector $\mathbf{u}_{n}(\mathbf{p} ; \mathbf{r}, t)$ is the total fields for an estimated parameter $\mathbf{p}$ due to the $n$th equivalent surface current $\mathbf{s}_{n}, n=1, \cdots, N$. Note that the surface current $\mathbf{s}_{n}$ is given by the total fields on $\partial \Omega$ for the object with true material parameters $\mathbf{p}^{\text {true }}=\left(\varepsilon_{r}^{\text {true }}, \mu_{r}^{\text {true }}, \eta \sigma^{\text {true }}\right)$ due to the $n$th illuminating source $\mathbf{j}_{n}$. In practice, $\mathbf{s}_{n}$ is determined by the total fields measured on the observation surface $\partial \Omega$. In numerical simulations, $\mathbf{s}_{n}$ is synthesized directly by $\mathbf{j}_{n}$.

In order to confirm the effectiveness of the proposed method, let us examine, for simplicity's sake, a two dimensional nondestructive testing problem of retrieving the location and size of two unknown defects in a known host medium as shown in Fig. 2. The total field $\mathbf{u}(\mathbf{r}, t)$ is calculated by the finite-difference time-domain (FDTD) method and a genetic algorithm [19] is used to minimize the cost functional $Q(\mathbf{p})$. The homogeneous host medium with the relative permittivity $\varepsilon_{r}=4.0$ is a square of $2 \lambda \times 2 \lambda$ whose center is located at the origin $(x, y)=(0,0)$. Here, $\lambda$ is the wavelength in free space corresponding to 


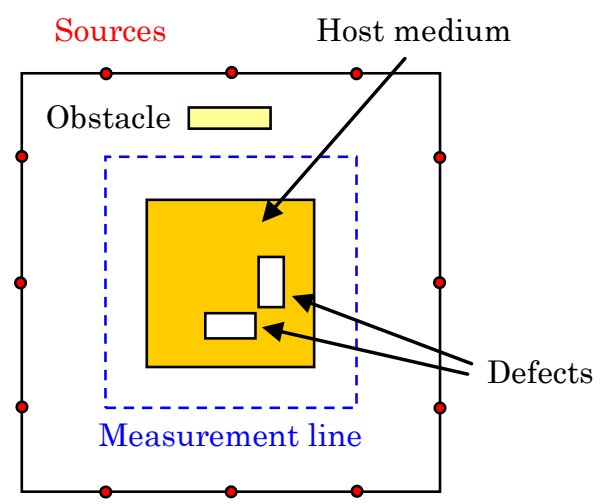

Fig. 2. Detection of two defects in a square cylinder

the highest frequency contained in the primary source (i.e., the frequency at which the amplitude spectrum of the source pulse becomes $5 \%$ of its maximum value):

$$
\mathbf{j}_{n}=I(t) \delta\left(\mathbf{r}-\mathbf{r}_{n}\right) \hat{z}
$$

where $\mathbf{r}=\mathbf{r}_{n}$ is the location of the $n$th primary source and the time factor

$$
I(t)=\frac{d^{3}}{d t^{3}} \exp \left[-\alpha^{2}(t-\tau)^{2}\right]
$$

and where $\tau=\beta \Delta t, \alpha=5.6 / \tau$, and $\beta=132$ with the time step size $\Delta t=0.98(\Delta x / c \sqrt{2})$ and the cell size $\Delta x=\Delta y=\lambda / 10 \sqrt{4}$. The defect 1 is a $0.3 \lambda \times 0.6 \lambda$ square of $\varepsilon_{r}=1.0$ with its centre at $(0.5 \lambda, 0)$ and the defect 2 is a $0.6 \lambda \times 0.3 \lambda$ square of $\varepsilon_{r}=1.0$ with its centre at $(0,-0.5 \lambda)$. The measurements are performed along the periphery $\partial \Omega$ of the square of side $3 \lambda$. The primary sources are located $\lambda$ away from the measurement line $\partial \Omega$. The background is free space.

The parameters characterizing host medium (the location, size, and dielectric constant), and the number and the dielectric constant of the defects are assumed to be known, while the location $\left(x_{c 1}, y_{c 1}\right)$ of the center and the size $l_{x 1} \times l_{y 1}$ of the defect 1 and the location $\left(x_{c 2}, y_{c 2}\right)$ of the center and the size $l_{x 2} \times l_{y 2}$ of the defect 2 are searched for. In order to show the estimation is not affected by clutter, a $\lambda \times 0.3 \lambda$ dielectric square obstacle with $\varepsilon_{r}=2.0$ is placed between the primary source and the observation surface. The information on the blocking square as well as the primary sources is used for calculating synthetic measurement data, while such information is not necessary in the inversion procedure of the proposed method. For the use of 12 successive illuminations, the best estimated values of the characterizing parameters in 10 runs of the GA search after 200 generations are the same values of true ones, $\left(x_{c 1}, y_{c 1}\right)=(0.50 \lambda, 0.00 \lambda)$, $l_{x 1} \times l_{y 1}=0.30 \lambda \times 0.60 \lambda \quad, \quad\left(x_{c 2}, y_{c 2}\right)=(0.00 \lambda,-0.50 \lambda) \quad, \quad$ and $l_{x 2} \times l_{y 2}=0.60 \lambda \times 0.30 \lambda$. The successful estimation demonstrates the effectiveness of the present approach. Interestingly, for a single illumination by any of the 12 sources, satisfactory results were also obtained.
In conclusion, we have presented an inverse scattering approach for reconstructing material parameters of an object of interest which does not require explicitly a-priori knowledge of incident fields. This indicates that even in the presence of any obstacle (know or unknown) near the primary source we do not need to calculate the scattered field by the obstacle if it locates outside the region enclosed by the observation surface. The sum of the field generated by the primary source and the scattered field by the obstacle is regarded as the incident field. The measured total fields consist of the incident field and the scattered field by the object of interest. The material parameters of the object are estimated from the measured total field data only. We close with three remarks. The proposed approach can be applied to whatever temporal regime and the time-dependence used in the numerical examples is just for this presentation. In order to avoid computational complexity growing rapidly with the number of unknowns when using GAs, gradient-based optimization procedures are preferable. In practice a finite number of measurement points are used. We must examine how sparsely the measurement points can be located to get satisfactory reconstruction results. These issues will be considered in future studies.

\section{References}

1. W. C. Chew and Y. M. Wang, IEEE Trans. Med. Imag. 9, 218 (1990).

2. T. Harada, D. J. N. Wall, T. Takenaka, and T. Tanaka, IEEE Trans. Antennas Propag. 43, 784 (1995).

3. A. Franchois and C. Pichot, IEEE Trans. Antennas Propag. 45, 203 (1997).

4. T. Isernia, V. Pascazio, and R. Pierri, IEEE Trans. Geosci. Remote Sens. 35, 910 (1997).

5. S. Caorsi, A. Massa, and M. Pastorino, IEEE Trans. Microwave Theory Tech. 48, 1815 (2000).

6. L. Crocco, M. D' Urso, T. Isernia, Opt. Express 15, 3804 (2007).

7. M. D' Urso, K. Belkebir, L. Crocco, A. Litman, and T. Isernia, J. Opt. Soc. Am. A 25, 271 (2008).

8. P. Rocca, M. Benedetti, M. Donelli, D. Franceschini, and A. Massa, Inverse Probl. 25, 1 (2009).

9. M. Pastorino, Microwave Imaging, John Wiley, Hoboken, N.J. (2010).

10. L. Pan, X. Chen, Y. Zhong, and S. P. Yeo, J. Opt. Soc. Am. A 27, 2208 (2010).

11. G. Oliveri, Y. Zhong, X. Chen, and A. Massa, J. Opt. Soc. Am. A 28, 2057 (2011).

12. M. Moghaddam and W. C. Chew, IEEE Trans. Antennas Propag. 41, 177 (1993).

13. S. He, P. Fuks, and G. W. Larson, IEEE Trans. Antennas Propag. 44, 1277 (1996).

14. I. T. Rekanos, J. Electromagn. Waves Appl., 17, 271 (2003).

15. T. Takenaka, H. Zhou, and T. Tanaka, J. Opt. Soc. Am. A 20, 1867 (2003).

16. C.-H. Huang, C.-C. Chiu, C.-L. Li, and K.-C. Chen, PIER 82, 381 (2008).

17. M. Gustafsson and S. He, Radio Sci. 35, 525 (2000).

18. R. F. Harrington, Time-Harmonic Electromagnetic Fields, Wiley-IEEE Press; 2nd ed. (2001).

19. T. Moriyama, Z.-Q. Meng, and T. Takenaka, Microwave Opt. Thechnol. Lett. 53, 438 (2011). 